\title{
Moderation of the Association between Media Exposure and Youth Smoking Onset: Race/Ethnicity, and Parent Smoking
}

\author{
Susanne E. Tanski • Mike Stoolmiller • Meg Gerrard • \\ James D. Sargent
}

Published online: 8 September 2011

(C) The Author(s) 2011. This article is published with open access at Springerlink.com

\begin{abstract}
This study of youth smoking onset aims to replicate previously published media moderation effects for race/ethnicity in a national longitudinal multiethnic sample of U.S. adolescents. Previous research has demonstrated that associations between media and smoking during adolescence are greater for Whites than Hispanics or Blacks, and for youth living in non-smoking families. In this study, changes in smoking status over 24 months were assessed among 4,511 baseline never-smokers. The incidence of smoking onset was $14.3 \%$ by 24 months with no differences by race/ethnicity. Blacks had higher exposure to movie smoking and overall television viewing compared with Whites and Hispanics. Whites responded to movie smoking regardless of parent smoking but more strongly if their parents were non-smokers. In contrast, Black adolescents
\end{abstract}

This work was supported by National Cancer Institute grant CA77026 and the American Legacy Foundation.

Electronic supplementary material The online version of this article (doi:10.1007/s11121-011-0244-3) contains supplementary material, which is available to authorized users.

S. E. Tanski · J. D. Sargent

Department of Pediatrics, Dartmouth Medical School, Hanover, NH, USA

S. E. Tanski $\cdot$ M. Gerrard · J. D. Sargent

Norris Cotton Cancer Center,

Lebanon, NH, USA

M. Stoolmiller

Oregon Social Learning Center, University of Oregon,

Eugene, OR, USA

\section{S. E. Tanski $(\bowtie)$}

Pediatrics, Dartmouth-Hitchcock Medical Center,

One Medical Center Drive, Hinman, Box 7925, Lebanon,

NH 03756, USA

e-mail: Susanne.e.tanski@dartmouth.edu showed little behavioral response to any media, regardless of parent smoking. Hispanic adolescents responded only to TV viewing and only when their parents did not smoke. In an analysis assessing the influence of the race of smoking characters on smoking behavior of White and Black adolescents, Whites responded to both White and Black movie character smoking, whereas Blacks responded only to smoking by Black movie characters. Taken as a whole, the findings replicate and extend previous findings, suggesting media factors are more influential among adolescents at low to moderate overall risk for smoking. We draw analogies between these lowmoderate risk adolescents and "swing voters" in national elections, suggesting that media effects are more apt to influence an adolescent in the middle of the risk spectrum, compared with his peers at either end of it.

Keywords Smoking initiation - Media exposure ·

Adolescent behavior $\cdot$ Racial differences

\section{Introduction}

Youth smoking continues to be a significant concern worldwide. Exposure to smoking in the movies has been linked with smoking initiation among adolescents in several regional longitudinal adolescent samples: three in the US (Dalton et al. 2003; Distefan et al. 2004; Titus-Ernstoff et al. 2008) and one in Germany (Hanewinkel and Sargent 2008); and with current smoking (within the past 30 days) in a Mexican study (Thrasher et al. 2009). The robust association between exposure to movie smoking and behavior has been interpreted as evidence of a causal effect in one recent review, conducted by the National Cancer Institute (2008). This causal statement has been followed by 
research demonstrating a decline in movie smoking that coincides with recent declines in adolescent smoking in the United States (Sargent and Heatherton 2009). Thus, research that examines how, why, and for whom these important media effects occur could have implications on youth smoking.

This manuscript focuses on factors that moderate the association between movie smoking and adolescent smoking onset. Exploring moderation factors, particularly those factors that replicate from study-to-study, can enhance our understanding of how media exerts its influence and for whom media influence is important. One moderation factor that has been examined is race/ethnicity. The majority of movie stars and movie smokers are white and upper class (Dalton et al. 2002; Dozier et al. 2005; Hazan and Lipton 1994; Tanski et al. 2009; Worth et al. 2007); exploring moderation by race/ethnicity assesses the extent to which the predominantly White role models in movies may influence youth of other race/ethnicity. The association between movie exposure and smoking has been found to differ by Black versus White race in one longitudinal study such that Black youth were relatively non-responsive to movie exposure as compared to Whites (Jackson et al. 2007). (See also Gibbons et al. 2010 for a similar pattern of results for the effects of media exposure to alcohol use.) A longitudinal study examining the association between movie smoking exposure and smoking for MexicanAmerican adolescents found a weaker association for Mexican-American youth than that previously found for Whites (Wilkinson et al. 2009), while a different longitudinal study of Mexican adolescents found an association only for 30-day smoking, but not for smoking onset (Thrasher et al. 2009). In aggregate, these studies suggest weaker entertainment media effects among minority adolescents. These racial and ethnic differences raise the question of whether Black and, to a lesser extent, Hispanic adolescents process the predominantly White actor smoking they see in movies differently from White adolescents. By separating movie smoking exposure into White and Black actor smoking, this study begins to explore these issues.

Other studies have reported additional moderation effects for the movie smoking-adolescent smoking relation. For example, there is a stronger movie influence on initiation among children whose parents do not smoke (Dalton et al. 2003; Hanewinkel and Sargent 2008), and a stronger response to movie smoking in progression to established smoking among adolescents lower in sensation seeking (as compared to those high is sensation seeking) (Sargent et al. 2007). Another study has examined moderation of the movie smoking-behavior relation by team sports participation, finding that team sports participants (but not nonparticipants) responded to movie smoking (Adachi-Mejia et al. 2009). The commonality for each of these moderation effects (parent smoking, sensation seeking, or extracurricular sports involvement) is that the movie smoking/ behavior relation is stronger within the groups considered at lower risk for smoking (low sensation seeking, nonsmoking parents, etc). In previous articles, we have suggested that these interaction patterns argue against an unmeasured risk factor that correlates with movie smoking and adolescent smoking in such a way as to confound the movie smoking effect (Sargent et al. 2007). What has not been addressed, however, is whether these moderation patterns point to a general conclusion or theory about the circumstances under which media exposures exert influence on behavior.

The intent of this report is to evaluate racial differences in the association between media exposures and smoking initiation in a multi-ethnic longitudinal observational study of U.S. adolescents, with specific attention to previously reported moderation effects for race/ethnicity, parent smoking, sensation seeking, and extracurricular activities. We focus on exposure to movie smoking and weekday television viewing as media influences. Additionally, we conduct an analysis for White and Black adolescents to determine whether they respond differently to exposure to White and Black movie character smoking.

\section{Methods}

A detailed description of the recruitment methods for study participants has been published previously (Sargent et al. 2005). Briefly, between June and October 2003, a randomdigit dialed telephone survey recruited 6,522 US adolescents aged 10-14 years. Compared with the 2000 U.S. Census, the sample was nationally representative by age, sex, household income and region, but had higher percentages of Hispanics and slightly lower percentages of Blacks. Race was determined at the time of enrollment based on parent report. Parents could respond yes/no to multiple race and ethnicity questions. For these analyses, "White" comprises those who responded yes only to White race, and "Black" comprises those who responded yes only to Black race. All respondents reporting Hispanic ethnicity were categorized as Hispanic. All others (e.g., Asian, Native American) and those who identified themselves as multiple races were categorized as "other" race because their numbers were too small to break them out separately. Parental consent and adolescent assent were obtained prior to each phone interview, and all sensitive responses were captured using the telephone keypad to increase confidentiality. The survey cooperation rate was 0.66 , and our most conservative estimate of the response rate-one that estimates the number of eligible households among nonresponders, the Council of American Survey Research Organizations 
(CASRO) response rate-was 32\% (6,522 interviewed adolescents / an estimated 20,436 eligible households). All aspects of the survey were approved by the institutional review boards at Dartmouth Medical School and the survey research firm Westat (Rockville, MD). Given the study aim to examine the effect of movie smoking exposure on smoking uptake by White, Black and Hispanic adolescents, these analyses exclude 559 "multi-racial" or "other" adolescents. Analyses were conducted in 2009.

Follow-up Surveys Three follow-up surveys were conducted at 8-month intervals, with smoking initiation ascertained at the $8 \mathrm{M}, 16 \mathrm{M}$, and $24 \mathrm{M}$ surveys. The flow of subjects for the hazard analysis is shown in the Appendix Figure. At each survey point, the hazard analysis risk set " $N$ " was always smaller than the participation " $N$," which included ever-smokers and those who were subsequently lost to follow-up. Adolescents lost to follow-up at any point from baseline to the $24 \mathrm{M}$ survey watched more movies per week; had higher movie smoking exposure; were more likely to have parents and/or friends who smoked; were more likely to be non-White; were less likely to have excellent school performance; and had parents with lower educational attainment. Baseline smoking status did not predict the overall hazard of attrition or at any individual follow-up. Potential attrition bias was dealt with via multiple imputation as detailed below.

Behavioral Outcome Assessment of smoking initiation was based on the question, "How many cigarettes have you smoked in your life?" Smoking initiation was defined as having tried a few puffs or more.

Exposure Measurement Adolescents' exposure to movie smoking was estimated using the Beach method (Sargent et al. 2008), in which exposure to the top 100 U.S. box-office hits of the 5 years preceding the baseline survey (19982002, $\mathrm{N}=500$ ), and 32 movies that earned $>\$ 15$ million in gross box-office revenues during the first 4 months of 2003 was assessed. Respondents were asked whether they had ever seen each movie title from a unique list of movie titles, randomly selected from the larger pool. The movies were content coded for smoking. Based on the movies the adolescent had seen and the amount of smoking in each, a continuous exposure measure was created that estimated exposure to all 532 movies. The content coding reliability and this method of exposure assessment have been extensively validated (Sargent et al. 2008). We have previously shown that Black youth view more movies overall than White youth, and particularly more violent films (Worth et al. 2008).

In a second analysis, exposure to smoking by White and Black major characters separately was examined among
White and Black adolescents, with the hypothesis that adolescents would be more responsive to movie character smoking by actors of the same race as the individual subject ("race-matched"). This analysis excluded Hispanic adolescents, as there is little movie exposure to Hispanic smoking (only $3.2 \%$ of major movie characters are Hispanic). The method mirrored that used in a previous publication to parse movie smoking effects by character valence ("good guy" vs. "bad guy") (Tanski et al. 2009). A major character was one that the content coder deemed essential to the plot of the movie (median of 6 [interquartile range 5-8] major characters per movie). Coders determined race/ethnicity for each major character and counted episodes of smoking. The exposure was divided into two categories: smoking by Black major characters (representing 13.9\%) and smoking by all others. As White characters predominate the category of all others, hereafter this group will be referred to as White character smoking. Major character smoking exposure is based on actual episodes viewed by movie, and is not scaled to represent exposure to the larger pool of 532 movies from which the individual's unique random movie lists were drawn.

Covariates Based on results from previous studies of adolescent smoking (Sargent et al. 2001, 2005), a broad range of covariates was included to control for possible confounding (see Appendix Table 1 for details). Covariates included age, gender, race, parental education, school performance, participation in extracurricular activities, parent, sibling and peer smoking, and overall quantity of TV viewing. Television content was not assessed. We controlled for sensation seeking (Zuckerman et al. 1972) using a measure validated for young adolescents (Sargent et al. 2010) and for parenting style using a measure validated by Jackson and colleagues (1994).

Statistical Analysis The available data consist of a baseline survey and three follow-up assessments, allowing determination of smoking onset at the $8 \mathrm{M}, 16 \mathrm{M}$, and $24 \mathrm{M}$ surveys. An incident case was defined as an adolescent who became a smoker from the pool of those who were non-smokers at the previous survey. The multivariate association between exposure to smoking in movies and the hazard of smoking onset at each time point was assessed using discrete time hazard regression to estimate multivariate hazard ratios for time to event occurrence (Allison 1984; Muthen and Masyn 2005; Singer and Willett 2003) and are interpreted similarly to odds ratios. Participants were censored after they tried smoking. Participants who never tried smoking were censored at the final time interval, and participants who dropped out were censored at their last survey. Censoring was assumed to be independent of the hazard of initiating smoking, conditional on covariates included in the model 
(Schafer and Graham 2002). In order to directly compare covariates, continuous covariates were all scaled such that zero corresponded to the $5^{\text {th }}$ percentile and 1 to the $95^{\text {th }}$ percentile for their distributions; extreme values in either direction were recoded to 0 or 1 . Ordered categorical predictors were rescaled similarly so that the lowest value was 0 , the highest value was 1 and intermediate values spaced evenly in between. These transformations allowed for better comparison of the effect sizes between continuous, ordered categorical and dichotomous variables and also limited the influence of outliers for skewed continuous variables. With regard to attrition, the MICE procedure in $\mathrm{R}$ was used to stochastically impute missing data (van Buuren and Groothuis-Oudshoorn in press). To improve the quality of the imputations, a number of baseline auxiliary variables were included only in the imputation model that were predictive of missing data but not necessarily the outcome. All variables were treated as numeric and the predictive mean matching procedure was used to create 20 imputed values for each missing score. Convergence was assessed by checking plots of the mean and variance of the imputations for each variable across the 20 streams for signs of problems such as trends or lack of proper mixing. No problems were apparent. For descriptive statistics, we averaged across the 20 imputations to get a single best estimate for each missing data point.

Our main interest was in testing a previously reported interaction between race/ethnicity and media (movies and television) exposure (Jackson et al. 2007). However, based on previous studies of predominantly White samples, two variables were considered as candidates for three-way interaction effects - parent smoking (Dalton et al. 2003; Hanewinkel and Sargent 2008) and sensation seeking (Sargent et al. 2007); these were assessed in a set of three-way interactions between the variable, other covariates, and race/ethnicity. In preliminary analyses, parent smoking was found to be an important three-way interaction variable with respect to media exposures, but sensation seeking was not, so it was not considered further for moderation involving media effects. Additionally, we checked for significant (at $p<0.05$ ) differences in other predictor effects across ethnic groups, and then constrained effects to be equal when not significantly different (Cohen and Cohen 1983).

\section{Results}

Exposures and Behavior by Race and Ethnicity Table 1 shows how media exposure variables and covariates varied by race/ethnicity, the primary moderation variable in this analysis. Exposure to movie smoking varied by race/ ethnicity, with Black adolescents seeing significantly more movie smoking than Whites or Hispanics. Quantity of weekday television viewing was higher among Blacks than Hispanics, and higher among Hispanics than Whites. Reports of friend smoking were equal across race/ethnicity, but Hispanics were less likely than Whites or Blacks to have a parent or sibling who smoked. Other covariate differences are noted in Table 1. Rates of smoking initiation did not differ markedly by race/ethnicity, varying from 5.9 to $8 \%$ for each 8 -month period (Table 1).

Moderation Effects The unadjusted effect of exposure to movie smoking on smoking initiation differed by race/ ethnicity, as illustrated in Fig. 1, with the unadjusted direct relation between the odds of trying smoking and movie smoking exposure being positive and significant for all three groups (White hazard=7.6, Black hazard=2.2, Hispanic hazard=3.7). The relation was significantly stronger for Whites vs. Hispanics $(p=.007)$ and Blacks $(p<.001)$, but not significantly different for Hispanics vs. Blacks $(p=.19)$.

Multivariate adjusted interactions between parent smoking status, media exposure and race/ethnicity are shown in Table 2. With regards to movie smoking among adolescents whose parents did not smoke, exposure to movie smoking strongly predicted smoking initiation for Whites (hazard estimate 4.6 [3.0, 76.9]), but not for Hispanics (1.6 [0.9, $3.1])$ and Blacks $(1.1[0.4,2.7])$. For adolescents whose parents did smoke, movie smoking predicted trying smoking only for Whites $(1.8[1.1,2.8])$, but the relation was significantly and substantially weaker than for Whites whose parents did not smoke. With regard to weekday television viewing among adolescents whose parents did not smoke, exposure to higher levels of television predicted smoking initiation for Hispanics only. For adolescents whose parents did smoke, the hazard estimates for television viewing were roughly equivalent across race/ ethnicity groups.

Effect of White and Black Movie Character Smoking on White and Black Adolescents As mentioned, this analysis excluded Hispanic adolescents because there were so few Hispanic characters and representative smoking episodes. While there are more Black than Hispanic characters (Black characters represent about $11 \%$ of all characters), only about $17 \%$ of them smoke. Thus, there is a four-fold difference in the possible movie smoking exposure from White characters (maximum 120 episodes) than from Black characters (maximum 30 episodes). Based on the movies they reported seeing, Black adolescents had significantly higher exposure to movie smoking from White characters (median 25, interquartile range $[11,44]$ ) and Black characters $(3[1,9])$ compared with White adolescents $(19$ [7,38] and $0[0,3]$ for White and Black characters, respectively). 
Table 1 Risk/protective factors and tried smoking incidence by race/ethnicity

\begin{tabular}{|c|c|c|c|c|c|}
\hline & & \multicolumn{3}{|c|}{ Race/ethnicity } & \multirow[t]{2}{*}{ Significant Contrast } \\
\hline & & White & Black & Hispanic & \\
\hline \multicolumn{6}{|l|}{ Media Exposures } \\
\hline Movie smoking $[\operatorname{mean}(S D)]$ for 0 to 1 scale & & $.30(.27)$ & $.39(.29)$ & $.31(.28)$ & $\mathrm{H}, \mathrm{W}<\mathrm{B}$ \\
\hline \multirow[t]{6}{*}{ Weekday television viewing } & & & & & $\mathrm{W}<\mathrm{H}<\mathrm{B}$ \\
\hline & None & $5.9 \%$ & $6.6 \%$ & $4.3 \%$ & \\
\hline & $<1 \mathrm{~h}$ & $22.0 \%$ & $9.2 \%$ & $17.2 \%$ & \\
\hline & $1-2 \mathrm{~h}$ & $49.5 \%$ & $35.4 \%$ & $48.6 \%$ & \\
\hline & $3-4 \mathrm{~h}$ & $16.8 \%$ & $27.3 \%$ & $23.1 \%$ & \\
\hline & $>4 \mathrm{~h}$ & $5.8 \%$ & $21.5 \%$ & $6.7 \%$ & \\
\hline Has TV in the bedroom & & $52.2 \%$ & $78.3 \%$ & $65.8 \%$ & $\mathrm{~W}<\mathrm{H}<\mathrm{B}$ \\
\hline \multicolumn{6}{|l|}{ Socio-demographics } \\
\hline \multirow[t]{4}{*}{ Parent education } & & & & & $\mathrm{H}<\mathrm{B}<\mathrm{W}$ \\
\hline & HS grad or less & $27.9 \%$ & $45.0 \%$ & $67.6 \%$ & \\
\hline & Associate's & $31.3 \%$ & $33.9 \%$ & $19.0 \%$ & \\
\hline & $>=$ Bachelor's & $40.8 \%$ & $21.1 \%$ & $13.5 \%$ & \\
\hline \multicolumn{6}{|l|}{ Social influences and school } \\
\hline Friend(s) smoke & & $15.1 \%$ & $17.3 \%$ & $16.9 \%$ & None \\
\hline Sibling(s) smoke & & $12.2 \%$ & $12.9 \%$ & $9.2 \%$ & $\mathrm{H}<\mathrm{B}, \mathrm{W}$ \\
\hline Parent(s) smoke & & $30.2 \%$ & $32.3 \%$ & $25.0 \%$ & $\mathrm{H}<\mathrm{B}, \mathrm{W}$ \\
\hline Parenting style & & $.60(.27)$ & $.61(.28)$ & $.60(.29)$ & None \\
\hline School performance average or below average & & $63.3 \%$ & $73.7 \%$ & $74.4 \%$ & $\mathrm{~W}<\mathrm{B}, \mathrm{H}$ \\
\hline Extracurricular activities & & $.50(.27)$ & $.51(.29)$ & $.45(.29)$ & $\mathrm{H}<\mathrm{B}, \mathrm{W}$ \\
\hline \multicolumn{6}{|l|}{ Characteristics of the adolescent } \\
\hline Sensation seeking & & $.34(.28)$ & $.36(.27)$ & $.33(.28)$ & None \\
\hline Rebelliousness & & $.23(.28)$ & $.30(.30)$ & $.25(.30)$ & $\mathrm{H}, \mathrm{W}<\mathrm{B}$ \\
\hline \multicolumn{6}{|l|}{ Smoking onset } \\
\hline Baseline $\rightarrow 8 \mathrm{M}$ & $7.1 \%$ overall & $6.2 \%$ & $8.5 \%$ & $7.6 \%$ & None \\
\hline $8 \mathrm{~m} \rightarrow 16 \mathrm{M}$ & $6.8 \%$ overall & $6.7 \%$ & $7.8 \%$ & $8.0 \%$ & None \\
\hline $16 \mathrm{~m} \rightarrow 24 \mathrm{M}$ & $6.4 \%$ overall & $5.9 \%$ & $6.9 \%$ & $7.7 \%$ & None \\
\hline Baseline $\rightarrow 24 \mathrm{M}$ & $14.3 \%$ overall & $14.0 \%$ & $14.1 \%$ & $15.8 \%$ & None \\
\hline
\end{tabular}

Significance is presented based upon differences in means by race. In the first row, for example, $\mathrm{H}, \mathrm{W}<\mathrm{B}$ would be interpreted as there being no statistically significant difference in the prevalence of movie smoking between White and Hispanic youth, but both groups having less exposure than Black youth. All continuous variables were scaled so that 0 represents the 5th percentile and 1 represents the 95 th percentile for the distribution, values below 0 were set to 0 , and values above 1 were set to 1 ; thus, range was always $0-1$ for these variables. Means and $S D$ s presented for continuous variables and percentages for ordered categorical or dichotomous variables. Significance tests for race/ethnicity contrasts based on $\mathrm{t}$ tests for continuous variables, ordered logistic regression for ordered categorical variables and logistic regression for dichotomous variables

In line with results from the previous model in Table 2, we hypothesized that White adolescents would be sensitive to movie character smoking regardless of actor ethnicity or parent smoking. In contrast, however, we hypothesized that Black adolescents would be sensitive to Black character smoking and only if neither parent smoked. Conversely, we did not expect Black adolescents to be sensitive to movie character smoking if their parents smoked or if the movie character was non-Black.

To test these hypotheses, all the same background covariates as in Table 2 were included in the model and we first estimated four separate effects for White teens (all combinations of parent smoking by movie character race) and the same four separate effects for Black teens. The joint Wald test that all four White effects and the single Black effect (for Black teens + non-smoking parents + Black movie characters) were equal was upheld (chi-square $=2.27$, $d f=4, p=.31$ ). The joint Wald test that the same five effects were all zero was rejected (chi-square $=24.43, d f=5, p$ $<.001)$. The joint Wald test that the remaining three effects for Black teens were equal was upheld (chi-square for equality $=1.53, d f=2, p=.53$ ), and that these three effects 


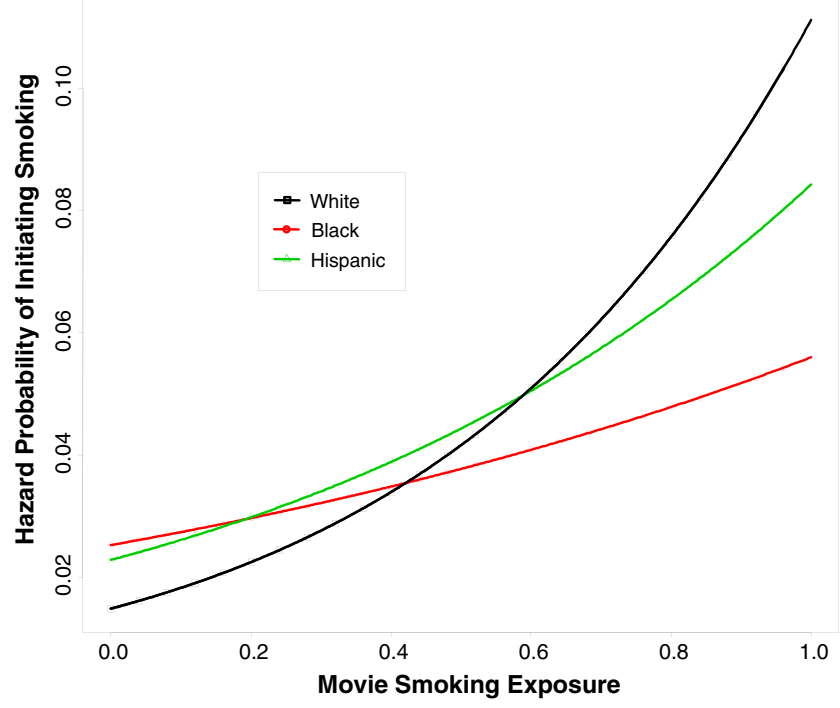

Fig. 1 Crude fitted probability of trying smoking as a function of exposure to movie smoking by race/ethnicity. For exposure (x-axis) zero and 1 correspond to the 6 th and $96^{\mathrm{h}}$ percentiles for exposure, respectively, and 0.24 corresponds to median exposure were zero was also upheld (chi-square $=2.16, d f=3, p=.54$ ). Finally, the Wald test that the average of the five positive effects was equal to the average of the three zero effects was rejected (chi-square $=9.79, d f=1, p<.001$ ).

We estimated a second model that incorporated the equality constraints implied by the joint tests. The movie character smoking effect for White adolescents and Blacks (+nonsmoking parents + Black character smoking) was significant $(b=.019, t=4.88, d f=594, p<.001)$ and the movie character smoking effect for Black teens exposed to non-Black movie characters or from families with parents that smoked was non-significant $(b=-.002$, $t=-.66, d f=578, p=.51)$. The Wald test that these two effects were equal was also rejected (chi-square $=16.43$, $d f=1, p<.001)$. Although the effect of movie character smoking per episode was the same for White and Black adolescents, the range of exposure $\left(5^{\text {th }}\right.$ to the $\left.95^{\text {th }}\right)$ for Whites is much larger, 1 to 97 episodes, then for Blacks, 0 to 13 episodes, primarily because White teens are susceptible to both Black and non-Black movie character smoking. Therefore, using the same approach as in Table 2 to standardize effect sizes, (i.e., the $5^{\text {th }}$ vs. the $95^{\text {th }}$

Table 2 Multivariate hazard model showing interaction effects

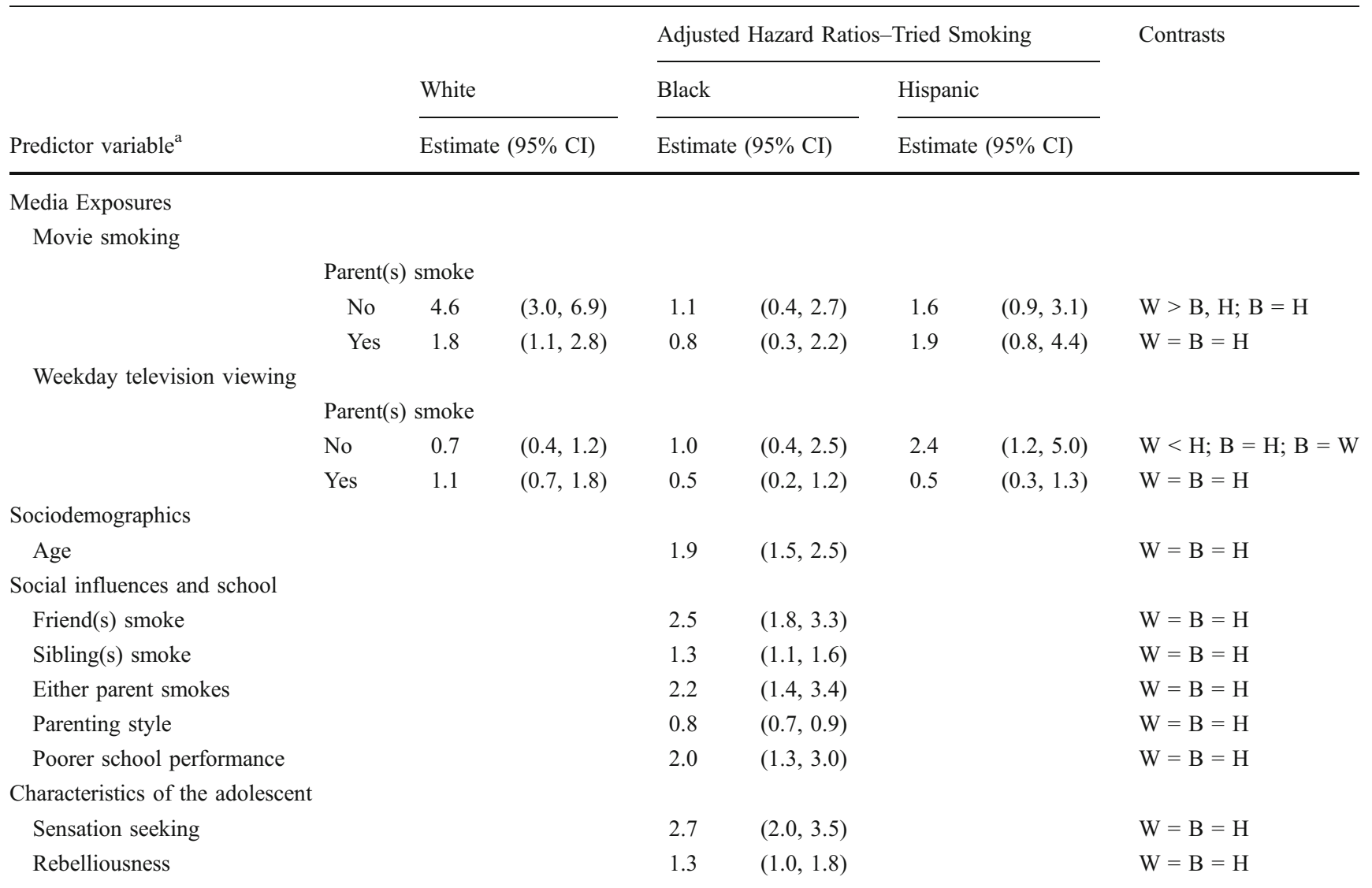

${ }^{a}$ Other variables not associated with trying smoking: parent education, adolescent gender, extracurricular activities and main effects for race/ ethnicity. Baseline hazard increases significantly at $16 \mathrm{M}$ and $24 \mathrm{M}$ compared to $8 \mathrm{M}$ 
percentile of the predictor) the adjusted hazard ratio for movie character smoking for White teens was 2.22, and for Blacks with non-smoking parents the hazard ratio was 1.28.

\section{Discussion}

The moderation analyses conducted on this are consistent with previous analyses, showing that the smoking behavior of Black (Jackson et al. 2007), and to a lesser extent, Hispanic (Wilkinson et al. 2009) adolescents is resistant to the influence of movie smoking as compared to Whites. These analyses also replicated expected moderation effects regarding parent smoking but not sensation seeking. However, when transition to established smoking was examined within this sample in a previous manuscript, a moderation effect was found for sensation seeking, with larger effects for low sensation seeking adolescents (Sargent et al. 2007).

This study extends previous studies in its examination of media effects by race and ethnicity by elucidating different media response patterns for White, Black and Hispanic adolescents in the context of whether parents smoke. White adolescents were responsive to movie smoking regardless of parental smoking status, albeit with significantly weaker responses when parents smoked. In contrast, Black adolescents had the highest exposure to movies and television but were relatively unresponsive to movie smoking, regardless of parent smoking status. Hispanic adolescents were somewhere in between, a modest reponse to movies that did not reach statistical significance and a somewhat stronger response to increased amounts of television, only when their parents did not smoke.

While Black youth appear resistant to movies' influence when measured in aggregate, the disaggregation of movie exposure by White and Black actors demonstrates an association between exposure to same-race actor smoking and adolescent smoking. The findings raise the possibility that racial and ethnic minorities are more responsive to seeing actors smoke when the actors are matched to their own race/ethnicity. It should also be noted that White adolescents responded to both White and Black character smoking, so the results should not be taken to suggest that there are no effects across racial and ethnic categories. An effect of Black actor smoking on White adolescents seems plausible, as Black culture often sets national trends, with Black role models impacting mainstream White culture. This is readily seen in the music industry: Hip-Hop music spawned a culture that went beyond music to influence dance and clothing, and continues to be popular with some groups of White male adolescents. The finding for Hispanics and television should prompt closer scrutiny of exposure to smoking on Spanish language television, where
Hispanic adolescents could be exposed to character smoking of their own ethnicity with higher frequency than would be possible in movies.

The moderation of media effects on parent smoking (Dalton et al. 2003; Hanewinkel and Sargent 2008), sensation seeking (Sargent et al. 2007; Stoolmiller et al. 2010), and extracurricular participation (Adachi-Mejia et al. 2009) supports previous suggestions that media effects are most apparent for adolescents generally considered at lower risk. This seems logical when one considers a population of youth as a "spectrum" of risk: One would not expect exposure to entertainment media to substantively alter risk for smoking of a high sensation-seeking adolescent whose parents and friends smoke - it is already quite high. At the other end of the risk spectrum, large doses of movie smoking are unlikely to prompt a highly motivated honors scholar-athlete whose parents and friends do not smoke to contemplate smoking. Instead, media exposure is more likely to affect those in the middle of the risk spectrum: adolescents who are relatively ambivalent about smoking; i.e., those who are not intending to smoke, but may be willing to under some circumstances (Gerrard et al. 2005). This is analogous to media effects on voters' decisions. Political campaigns direct their media messages to the "swing voter" (Mayer 2008) - the roughly $30 \%$ of the electorate that could vote either way because they see value in both candidates - because swing voters are more likely to change their vote in response to a media campaign than those at the extreme ends of the political spectrum. This concept may have implications for how we target adolescents for anti-smoking media campaigns. If studies confirm media effects are more pronounced for ambivalent (swing) adolescent non-smokers, these youth may be an appropriate target for anti-smoking advertising messages, rather than, for example, high sensation seeking youth who are already at high risk of smoking (Palmgreen et al. 2001; Sargent et al. 2010; Stephenson et al. 2002).

Development of a low-risk profile for smoking due to high resistance to smoking and hence, as we posit, a low susceptibility to media influence, is likely to be a multidimensional phenomenon and warrants further investigation. The influences and processes that produce ambivalent and prosmoking teens may be determined by distinctly different phenomena than the development of low-risk teens. We suggest that although a single, powerful risk factor (parent smoking or high sensation seeking) may be sufficient to attenuate the effect of media, the absence of that factor does not necessarily make all adolescents invulnerable. Instead, we suggest that certain factors may moderate risk for some groups of non-smokers and not for others; e.g., having low sensation seeking may serve as a buffer against movie exposure for adolescents who are generally resistant to smoking but not for the "swing" adolescents, those who are ambivalent about smoking or willing (but not intending) to smoke. Consistent 
with previous research on willingness to use substances (Gibbons et al. 2003), exposure to smoking in the media may have relatively little short-term impact on adolescents who are unwilling to consider smoking or those who intend to try smoking, but significant impact on adolescents who indicate a willingness to try smoking under some circumstances. Further research is needed to confirm if this group of willing non-smokers behaves like the swing votersusceptible to media messages-and can be targeted based on their willingness to smoke.

This study is strengthened by the use of a nationally representative sample, enhancing the external validity of the results, and the large sample size, which improves power to detect interactions by race/ethnicity. As with any study, there are several limitations that affect interpretation of the results. As with other longitudinal studies, this sample had higher rates of attrition among minorities and subjects at higher risk for adverse risk behaviors. Higher attrition in these groups could thus bias comparisons. However, within this sample the Black and Hispanic youth actually had slightly higher rates of smoking initiation over time, suggesting there was no bias against retention of minority adolescents that were at risk for trying smoking or the multiple imputation procedure eliminated potential attrition bias. The measure for television viewing was crude compared to the assessment of exposure to movies, so caution is warranted in interpreting the effect of television viewing on Hispanic youth. Self-reports of adolescent smoking are subject to recall and social desirability bias; however, several previous studies have found that adolescents accurately report smoking status when assured confidentiality (Murray et al. 1987; Murray and Perry 1987; Wills and Cleary 1997). Finally, although there were controls for many factors related to youth smoking, there may be an unmeasured confounding factor that accounts for some of the reported media effects.

In conclusion, using a multi-ethnic sample, this study replicates moderation effects for movie smoking on smoking initiation, provides an explanation for attenuated response among Black and Hispanic adolescents, and suggests a reason for negative moderation effects on several important smoking risk factors. Future studies should explore the hypothesis that media effects are more influential among adolescents in the middle of the risk spectrum, defining these youth through attitudinal mediators such as willingness to smoke, or through use of a multi-dimensional risk score for smoking propensity.

Open Access This article is distributed under the terms of the Creative Commons Attribution Noncommercial License which permits any noncommercial use, distribution, and reproduction in any medium, provided the original author(s) and source are credited.

\section{References}

Adachi-Mejia, A. M., Primack, B. A., Beach, M. L., Titus-Ernstoff, L., Longacre, M. R., Weiss, J. E., et al. (2009). Influence of movie smoking exposure and team sports participation on established smoking. Archives of Pediatrics \& Adolescent Medicine, 163, 638-643.

Allison, P. D. (1984). Event history analysis: Regression for longitudinal event data. Thousand Oaks, CA: Sage Publications.

Cohen, J., \& Cohen, P. (1983). Applied multiple regression/correlation analysis for the behavioral sciences. Hillsdale, NJ: Lawrence Erlbaum Associates.

Dalton, M. A., Sargent, J. D., Beach, M. L., Titus-Ernstoff, L., Gibson, J. J., Ahrens, M. B., et al. (2003). Effect of viewing smoking in movies on adolescent smoking initiation: A cohort study. Lancet, $362,281-285$.

Dalton, M. A., Tickle, J. J., Sargent, J. D., Beach, M. L., Ahrens, M. B., \& Heatherton, T. F. (2002). The incidence and context of tobacco use in popular movies from 1988 to 1997. Preventive Medicine, 34, 516-523.

Distefan, J. M., Pierce, J. P., \& Gilpin, E. A. (2004). Do favorite movie stars influence adolescent smoking initiation? American Journal of Public Health, 94, 1239-1244.

Dozier, D. M., Lauzen, M. M., Day, C. A., Payne, S. M., \& Tafoya, M. R. (2005). Leaders and elites: Portrayals of smoking in popular films. Tobacco Control, 14, 7-9.

Gerrard, M., Gibbons, F. X., Stock, M. L., Lune, L. S., \& Cleveland, M. J. (2005). Images of smokers and willingness to smoke among African American pre-adolescents: An application of the prototype/willingness model of adolescent health risk behavior to smoking initiation. Journal of Pediatric Psychology, 30, 305318.

Gibbons, F. X., Gerrard, M., \& Lane, D. J. (2003). A social-reaction model of adolescent health risk. In J. M. Suls \& K. A. Wallston (Eds.), Social psychological foundations of health and illness (pp. 107-136). Oxford: Blackwell.

Gibbons, F. X., Pomery, E. A., Gerrard, M., Sargent, J. D., Weng, C. Y., Wills, T. A., et al. (2010). Media as social influence: Racial differences in the effects of peers and media on adolescent alcohol cognitions and consumption. Psychology of Addictive Behaviors, 24, 649-659.

Hanewinkel, R., \& Sargent, J. D. (2008). Exposure to smoking in internationally distributed American movies and youth smoking in Germany: A cross-cultural cohort study. Pediatrics, 121, e108-e117.

Hazan, A. R., \& Lipton, H. L. (1994). Popular films do not reflect current tobacco use. American Journal of Public Health, 84, 998-1000.

Jackson, C., Bee-Gates, D. J., \& Henriksen, L. (1994). Authoritative parenting, child competencies, and initiation of cigarette smoking. Health Education Quarterly, 21, 103-116.

Jackson, C., Brown, J. D., \& L'Engle, K. L. (2007). R-rated movies, bedroom televisions, and initiation of smoking by white and black adolescents. Archives of Pediatrics \& Adolescent Medicine, $161,260-268$.

Mayer, W. G. (2008). The swing voter in American politics. Washington, DC: Brookings Institution Press.

Murray, D. M., O’Connell, C. M., Schmid, L. A., \& Perry, C. L. (1987). The validity of smoking self-reports by adolescents: A reexamination of the bogus pipeline procedure. Addictive Behaviors, 12, 7-15.

Murray, D. M., \& Perry, C. L. (1987). The measurement of substance use among adolescents: When is the 'bogus pipeline' method needed? Addictive Behaviors, 12, 225-233. 
Muthen, B., \& Masyn, K. (2005). Discrete-time survival mixture analysis. Journal of Educational and Behavioral Statistics, 30, 27-58.

National Cancer Institute. (2008). The role of the media in promoting and reducing tobacco use. Tobacco Control Monograph No. 19. Bethesda, MD: U.S. Department of Health and Human Services, National Institutes of Health, National Cancer Institute. NIH Pub. No. 07-6242.

Palmgreen, P., Donohew, L., Lorch, E. P., Hoyle, R. H., \& Stephenson, M. T. (2001). Television campaigns and adolescent marijuana use: Tests of sensation seeking targeting. American Journal of Public Health, 91, 292-296.

Sargent, J. D., Beach, M. L., Adachi-Mejia, A. M., Gibson, J. J., Titus-Ernstoff, L. T., Carusi, C. P., et al. (2005). Exposure to movie smoking: Its relation to smoking initiation among US adolescents. Pediatrics, 116, 1183-1191.

Sargent, J. D., Beach, M. L., Dalton, M. A., Mott, L. A., Tickle, J. J., Ahrens, M. B., et al. (2001). Effect of seeing tobacco use in films on trying smoking among adolescents: Cross sectional study. BMJ, 323, 1394-1397.

Sargent, J. D., \& Heatherton, T. F. (2009). Comparison of trends for adolescent smoking and smoking in movies, 1990-2007. JAMA, $301,2211-2213$

Sargent, J. D., Stoolmiller, M., Worth, K. A., Dal Cin, S., Wills, T. A., Gibbons, F. X., et al. (2007). Exposure to smoking depictions in movies: Its association with established adolescent smoking. Archives of Pediatrics \& Adolescent Medicine, 161, 849-856.

Sargent, J. D., Tanski, S., Stoolmiller, M., \& Hanewinkel, R. (2010). Using sensation seeking to target adolescents for substance use interventions. Addiction, 105, 506-514.

Sargent, J. D., Worth, K. A., Beach, M., Gerrard, M., \& Heatherton, T. F. (2008). Population-based assessment of exposure to risk behaviors in motion pictures. Communication Methods and Measures, 2, 134-151.

Schafer, J. L., \& Graham, J. W. (2002). Missing data: Our view of the state of the art. Psychological Methods, 7, 147-177.

Singer, J. D., \& Willett, J. B. (2003). Applied longitudinal data analysis: Modeling change and event occurence. New York: Oxford University Press.

Stephenson, M. T., Morgan, S. E., Lorch, E. P., Palmgreen, P., Donohew, L., \& Hoyle, R. H. (2002). Predictors of exposure from an antimarijuana media campaign: Outcome research assessing sensation seeking targeting. Health Communication, 14, 23-43.

Stoolmiller, M., Gerrard, M., Sargent, J. D., Worth, K. A., \& Gibbons, F. X. (2010). R-rated movie viewing, growth in sensation seeking and alcohol initiation: Reciprocal and moderation effects. Prevention Science, 11, 1-13.

Tanski, S. E., Stoolmiller, M., Dal Cin, S., Worth, K., Gibson, J., \& Sargent, J. D. (2009). Movie character smoking and adolescent smoking: Who matters more, good guys or bad guys? Pediatrics, 124, 135-143.

Thrasher, J. F., Sargent, J. D., Huang, L., Arillo-Santillan, E., Dorantes-Alonso, A., \& Perez-Hernandez, R. (2009). Does film smoking promote youth smoking in middle-income countries?: A longitudinal study among Mexican adolescents. Cancer Epidemiology, Biomarkers \& Prevention, 18, 3444-3450.

Titus-Ernstoff, L., Dalton, M. A., Adachi-Mejia, A. M., Longacre, M. R., \& Beach, M. L. (2008). Longitudinal study of viewing smoking in movies and initiation of smoking by children. Pediatrics, 121, 15-21.

van Buuren, S., \& Groothuis-Oudshoorn, K. (in press). MICE: Multivariate imputation by chained equations in R. Journal of Statistical Software.

Wilkinson, A. V., Spitz, M. R., Prokhorov, A. V., Bondy, M. L., Shete, S., $\&$ Sargent, J. D. (2009). Exposure to smoking imagery in the movies and experimenting with cigarettes among Mexican heritage youth. Cancer Epidemiology, Biomarkers \& Prevention, 18, 3435-3443.

Wills, T. A., \& Cleary, S. D. (1997). The validity of self-reports of smoking: Analyses by race/ethnicity in a school sample of urban adolescents. American Journal of Public Health, 87, 56-61.

Worth, K. A., Duke, J., Green, M. C., \& JD, S. (2007). Character smoking in top box office movies Legacy First Look Report p. 19. Washington, DC: American Legacy Foundation.

Worth, K. A., Gibson Chambers, J., Nassau, D. H., Rakhra, B. K., \& Sargent, J. D. (2008). Exposure of US adolescents to extremely violent movies. Pediatrics, 122, 306-312.

Zuckerman, M., Bone, R. N., Neary, R., Mangelsdorff, D., \& Brustman, B. (1972). What is the sensation seeker? Personality trait and experience correlates of the sensation-seeking scales. Journal of Consulting and Clinical Psychology, 39, 308-321. 